\title{
LA DIFERENCIA LÓGICA ENTRE LA FILOSOFÍA Y LA CIENCIA
}

El siguiente ensayo es un intento de determinar lógicamente la diferencia entre la filosofía y la ciencia. Aunque esto se ha hecho antes, por ejemplo en las obras de Kant y Cassirer, la distinción no se ha trazado con suficiente claridad como para evitar confusiones que hoy en día no solamente obstruyen el progreso de la filosofía, sino también el de la ciencia, conduciendo a una filosofía seudo-científica ${ }^{1}$ y una ciencia seudo-filosófica. ${ }^{2}$

\section{Dos tipos de sistemas}

Existe una diferencia obvia e inmediata entre los textos de filosofía y los textos, digamos, de física atómica. Cuando leemos un texto de ética, digamos de Aristóteles o de John Dewey, entendemos casi todo lo que éstos dicen. Su lenguaje es más o menos el de nuestra habla ordinaria; con sólo poder háblar y leer inteligentemente no tenemos mayor dificultad para seguirlos. Con el texto de física atómica ocurre algo muy diferente. Nuestro lenguaje cotidiano y nuestra inteligencia no nos sirven de nada. Hay en el texto muy pocos nombres habituales, y las letras del lenguaje están mezcladas con símbolos que son irreconocibles a menos que los hayamos aprendido mediante un esfuerzo especial y específico. El texto de física parece ser mucho más complejo y mucho más preciso que el de ética.

Esta diferencia debe darnos que pensar. Debemos preguntarnos cuál es la razón de esta diferencia. ¿Son los fenómenos de la vida humana de tal manera más simples y menos estructurados que los de la naturaleza que se les puede entender en un lenguaje tanto más simple y menos estructurado? ¿O es un texto de física más complejo y estructurado que lo necesario? Recordemos que la física aristotélica se escribió en la misma clase de lenguaje que la ética aristotélica, y que Galileo introdujo el lenguaje matemático que encontramos, grandemente desarrollado, en el texto de física atómica. ¿Es que entonces todo el desarrollo de la física moderna descansa sobre un error? ¿O es sencillamente que la ética se encuentra rezagada en su desarrollo? En vista del hecho de que las situaciones de la vida humana parecen ser cuando

1 V. gr. en la filosofía de John Dewey, quien propuso su enfoque teleológico en la teoría de los valores como un enfoque "científico".

2 V. gr. las diversas interpretaciones de la ecuación cuántica de Schrödinger ("onda de materia", "dualidad", "complementariedad", etc.), que son mutuamente exclusivas y, según Landé, fortuitas (Cf. Alfred Landé, Foundations of Quantum Theory, New Haven, 1957). 
menos tan complejas, y en realidad mucho más complejas, que las de la naturaleza, ¿no debería el lenguaje de la ética ser igualmente complejo y estructurado? $\mathrm{Y}$ el hecho, podríamos reflexionar, de que no lo sea y de que en la era de la física einsteiniana tengamos que recurrir a la ética aristotélica bien podría ser la razón de nuestra incapacidad para controlar la vida moralmente de la misma manera que controlamos la naturaleza físicamente.

Hay, desde luego, textos de ética más complejos y estructurados que los de Aristóteles y Dewey, por ejemplo, los de Kant y Spinoza. Pero aun éstos no desempeñan en la ética el papel que desempeñan los textos de física en esa ciencia. No vivimos de acuerdo con tal ética, de la misma manera que vivimos, por ejemplo, de acuerdo con la física de Einstein cuando disparamos un cohete atómico o con los conceptos de Maxwell y Edison cuando encendemos la luz, o con conceptos de la medicina cuando acudimos a un médico. ¿Quién, en una contigencia moral, ha consultado alguno de estos textos y hallado en ellos la solución de sus dificultades o ha acudido a un moralista? Los filósofos de la ética, como todos los otros filósofos excepto los de la naturaleza, viven en una torre de marfil, alejados de y sin la más ligera influencia sobre el desarrollo de los acontecimientos humanos que se supone deben ser orientados por ellos. La ética es todavía una filosofía, y no un método. Carece de la precisión, y por lo tanto de la complejidad, necesarias para poder ser aplicable a situaciones humanas definidas. Aun las éticas de Kant y Spinoza, pese a ser mucho más complejas y consistentes - y, en consecuencia, más difíciles de entender- que las de Aristóteles y Dewey, son todavía demasiado simples y demasiado inexactas $-\mathrm{y}$, en consecuencia, demasiado fáciles de entender - para poder ser aplicables a situaciones humanas de la misma manera que un texto de física atómica es aplicable a situaciones atómicas. Parece una paradoja el que un texto, para ser eficaz en la realidad cotidiana, deba ser incomprensible para las mentalidades cotidianas. Pero ése es el caso. Es como si el meollo de lo más concreto se hallase en lo más abstracto.

Tenemos, pues, diferentes grados de complejidades y, por lo tanto, de facilidad o de dificultad de los textos. La ética de Aristóteles es más fácil que la de Kant, y la ética de Kant es más fácil que la física de Einstein. Puesto que todos estos textos tienen por objeto explicarnos grandes regiones del mundo, la moral y la natural, estas diferencias significan algo, y en realidad algo de la más profunda importancia para la comprensión tanto del mundo moral como del natural.

Los textos en cuestión se distinguen por el grado de su sistematización. Aun cuando estas filosofías son "sistemáticas" hasta cierto grado, existe un abismo entre su carácter sistemático y el de un texto de Einstein o Dirac. Para poder entender la ética de Kant tenemos que conocer el sistema kantiano. Para poder entender la ética de Spinoza, tenemos que conocer el sistema de 
Spinoza. El conocimiento del sistema de Spinoza no nos ayuda esencialmente a entender la ética de Kant, ni el conocimiento del sistema de Kant nos ayuda esencialmente a entender la ética de Spinoza. Pero para poder entender los textos de Einstein o Dirac no tenemos que conocer los sistemas de Einstein o Dirac. Lo que tenemos que conocer es el sistema de las matemáticas. Y si conocemos ese sistema, podemos entender no sólo los textos de Einstein o Dirac, sino también cualquier otro texto de física o de cualquier ciencia natural exacta.

Existe, pues, una diferencia fundamental entre un sistema filosófico y un sistema científico. El sistema filosófico es un sistema único y singular que constituye la filosofía de un pensador individual. No existe ningún sistema filosófico objetivo y universal que ayude a comprender cualquier texto de ética o de filosofía en general. Pero sí existe un sistema objetivo y universal en las ciencias naturales, mediante el cual uno puede entender cualquier texto científico: el sistema de las matemáticas.

Es evidente que un sistema que comprende la totalidad de textos y argumentos en una región extensa del conocimiento, es un sistema más comprehensivo y significativo -un sistema más "sistemático", podríamos decir- que un "sistema" que comprende solamente unos cuantos textos y argumentos en una región limitada del conocimiento. Es evidente también que el sistema más comprehensivo debe ser más general y, al mismo tiempo, más específico -más abstracto y al mismo tiempo más concreto- que un sistema menos comprehensivo. Pues si un sistema ha de explicar los fenómenos en una región más extensa, debe ser más general que uno que explica los fenómenos en una región más reducida; y si há de explicar estos fenómenos debe ser más específico. Así, pues, nos encontramos nuevamente aquí con la abstracción en la concreción y la concreción en la abstracción que hemos observado antes.

Es característico de las matemáticas el ser al mismo tiempo abstractas en alto grado y profundamente concretas; y es esto lo que les da su eficacia en la vida real. La filosofía, y la ética en particular, carecen de semejante instrumento universal y preciso y, por lo tanto, carecen de eficacia real. Son "fáciles" y, por lo tanto, ineficaces. Están escritas en lenguaje cotidiano, aun cuando, como en el caso de Kant y de Spinoza, de una índole en cierto modo técnica. Pero estos "tecnicismos" filosóficos no son comparables con los de una ciencia exacta. 3 Términos físicos como "fuerza" o "masa" significan algo

3 A veces la "dificultad" de la filosofía no guarda proporción con su contenido. John Dewey, por ejemplo, tiene un mensaje muy sencillo, pero lo comunica de una manera extremadamente complicada. La física, en cambio, tiene que decir cosas extremadamente complejas, pero las dice de la manera más sencilla posible. Ásí, pues, si se considera la relación entre forma y contenido- la economía de la disciplina-, la mayor parte de la filosofía es innecesariamente difícil y la mayor parte de la ciencia razonablemente fácil. El hecho de que, a pesar de esto, la filosofía parezca fácil y la ciencia difícil, demuestra cuán infinitamente más complejo es lo que la ciencia tiene que decir y cuán infinitamente más ha diferenciado 
completamente diferente de lo que significan las mismas palabras en el lenguaje cotidiano o en la filosofía; en última instancia no significan más que ciertas fórmulas matemáticas. Esas mismas palabras en un texto de filosofía -a menos que se trate de un texto de filosofía de la ciencia- poseen más o menos sus significados cotidianos. ${ }^{4}$ Realmente, hay una moda filosófica que hace de tal significado la base de sus investigaciones; $y$, a despecho de algunos esfuerzos para distinguir "uso ideal" de "uso real", no resulta claro en qué sentido estos significados se distinguen de los del diccionario. ${ }^{5}$

Existen, luego, dos clases de "sistemas": los filosóficos y los científicos. Los primeros son "sistemas" individuales y particulares; no hay ningún sistema que los abarque a todos. Los segundos son todos ellos partes de un sistema abarcador, una superestructura o patrón universal que, en las ciencias naturales, son las matemáticas. Pero las matemáticas no constituyen el único sistema semejante posible, la única superestructura que determina a la ciencia exacta. Las ciencias musicales están estructuradas por la teoría de la armonía en sus "claves", "acordes", "escalas", etc. La estructura que determina la organización de los computadores electrónicos es la lógica simbólica. En general, hay una infinidad de tales superestructuras posibles, y sin alguna estructura formal de ese tipo una disciplina intelectual no puede ser una ciencia exacta. Si la ética, por ejemplo, ha de progresar para convertirse de filosofía en ciencia, es menester hallar o formular para ella una estructura formal de ese tipo. Siendo ése el caso, resulta importantísimo, naturalmente, entender en detalle la diferencia entre filosofía y ciencia, es decir, entre las dos clases de sistemas de los que hemos hablado.

\section{Conceptos analíticos y conceptos sintéticos}

La diferencia entre estas dos clases puede determinarse con precisión lógica: las dos clases de sistemas utilizan dos clases diferentes de conceptos. Los "sistemas" filosóficos utilizan conceptos analíticos, y los sistemas científicos

su tema o asunto en comparación con la filosofía; en una palabra, qué tipo infinitamente más avanzado de conocimiento es la ciencia.

${ }^{4}$ Como cuando John Dewey habla de las "fuerzas" que desintegran las viejas costumbres. Dewey-Tufts, Ethics, New York, 1942, pág. 113 et passim. La ética, para Dewey, no es "sino un planteamiento más consciente y sistemático del problema que ocupa la mente de cualquier persona... frente al conflicto moral". "La teoría moral no es sino una extensión de lo que está implicado en toda moralidad reflexiva." Op. cit., págs. $173 \mathrm{sig}$.

5 Estar "de acuerdo con el uso ordinario" se considera, en la discusión ética de hoy, como señal de que una teoría ética es adecuada y no inadecuada: todo lo contrario de lo que es el caso en la ciencia. (Cf. E. M. Adams, "'Ought' Again", en Philosophical Studies, VIII, diciembre de 1957, págs. 88 sig.; Philipp Frank, Philosophy of Science: The Link Between Science and Philosophy, Englewood Cliffs, New Jersey, 1957, Cap. 2; Modern Science and its Philosophy, New York, 1955, Cap. 7. También Wilhelm Dilthey, Das Wesen der Philosophie, en Gesammelte Schriften, V. Band, Leipzig und Berlin, 1924; págs. 414 sig. 
utilizan conceptos sintéticos. ${ }^{6}$ Los conceptos analíticos son conceptos cuyas comprehensiones consisten en predicados, en tanto que los conceptos sintéticos son conceptos cuyas comprehensiones consisten en relaciones formales. Esta diferencia lógica define al mismo tiempo la diferencia entre la filosofía y la ciencia. La filosofía y la ciencia guardan, pues, una relación lógica exacta; y la transición de una filosofía a una ciencia es un paso lógico definido. Este paso lo dio, en la filosofía natural, Galileo, quien efectuó la transición a la ciencia natural mediante una invención exacta y de gran alcance: sustituyó los predicados materiales de la filosofía aristotélica con relaciones formales -transformó el concepto aristotélico analítico del cambio en el concepto sintético de movimiento. El procedimiento exactamente análogo debe seguirse en la filosofía moral si es que ésta ha de transformarse en una ciencia: los conceptos analíticos "bueno", "valor", etc., deben transformarse en conceptos sintéticos.

Examinemos primero las diferencias entre estas dos clases de conceptos.

Un concepto es un contenido mental que tiene, como se ha dicho, "una doble vertiente". 7 Por una parte tiene significado o comprehensión, por otra parte tiene aplicabilidad o extensión. La comprehensión es un conjunto de palabras o de símbolos; la extensión es un conjunto de cosas, concretas o abstractas. Para tomar el ejemplo de un concepto analítico, el concepto "silla" tiene como comprehensión el grupo de palabras "estructura a la altura de las rodillas, con un asiento y un respaldo", y como extensión el conjunto (o la clase) de todas las sillas que son, han sido o serán. Las definiciones de los conceptos pueden ser comprehensivas o extensivas. Erf el Diccionario de la Real Academia Española la definición de "silla" es: "Asiento individual con respaldo y por lo general con cuatro patas." Esta es una definición comprehensiva. Si deseamos saber con mayor detalle qué es una silla, buscamos "asiento"..., pero quedamos decepcionados al leer: "Silla, banco u otra cosa destinada para sentarse en ella", lo cual no es la comprehensión sino la ex-

6 Esta es la terminología de Kant. La doctrina kantiana de los conceptos analíticos y sintéticos debe distinguirse de la de los juicios analíticos y sintéticos. La primera es lógica y está tratada en la Lógica de Kant. La segunda es epistemológica y está tratada en la Crítica de la Razón Pura. Los conceptos analíticos también han sido llamados sustanciales, materiales o abstractivos, y los conceptos sintéticos funcionales, formales o constructivos. La diferencia ha sido elaborada en los escritos de Ernst Cassirer, especialmente Substance and Function, Chicago, 1923. Generalmente no se la discute en los textos de lógica. Una excepción al respecto es David García Bacca, Introducción a la lógica moderna, Barcelona, 1936. Wilhelm Dilthey la ha utilizado sistemáticamente, en la terminología kantiana, para su fundamentación de las Geisteswissenschaften, especialmente en Ideen über eine beschreibende und zerglierdernde Psychologie, en Gesammelte Schriften, V. Band, Leipzig und Berlin, 1924; págs. 139-240. Para una discusión detallada de la distinción kantiana, véase Robert S. Hartman, "The Analytic and the Synthetic as Categories of Inquiry", en Perspectives in Philosophy, Ohio State University, 1953; págs. 55-78.

7 Daniel Christoff, "Le fondement logique des valeurs", en Proceedings of the Xth International Congress of Philosophy, Amsterdam, 1949; págs. 454 sig. 
tensión del concepto "asiento". Las definiciones del diccionario, de tal suerte, saltan de la comprehensión a la extensión, y viceversa. La comprehensión de "asiento" sería, por ejemplo: "Una superficie plena o curva destinada a alojar la prolongación inferior de la espina dorsal humana." Y esto, a su vez, podría ser determinado por la comprehensión de "superficie", "plana", "curva", "destinada", "alojar", etc. Así, en última instancia, todas las comprehensiones están conectadas. En un diccionario coherentemente comprehensivo podríamos ir "sistemáticamente" de una palabra a la otra hasta agotar el diccionario, sin que importe por cuál palabra comencemos. Todas las comprehensiones analíticas se implican las unas a las otras.

Esto es lo que hace posibles los "sistemas" analíticos. Tales sistemas no son más que cadenas de implicaciones de conceptos más o menos abstractos. La cadena de implicaciones se origina en la definición comprehensiva del concepto con el cual comenzamos, continúa con las definiciones comprehensivas de los conceptos contenidos en la primera definición -llamémoslas definiciones secundarias- $y$, por consiguiente, a las definiciones de los conceptos contenidos en las definiciones secundarias- llamémoslas definiciones terciarias- y así sucesivamente. De tal suerte la definición primaria en nuestro ejemplo es la de "silla": "una estructura a la altura de las rodillas, con un asiento y un respaldo". Las definiciones secundarias son las de "estructura", "altura de las rodillas", "asiento" y "respaldo", respectivamente. Las definiciones terciarias son las de los conceptos contenidos en las definiciones secundarias, por ejemplo, las de "asiento": "superficie", "plana", "curva", "destinada", etc. Este proceso de determinación cada vez más precisa de un concepto analítico puede continuar, claro está, ad infinitum -0 , mejor dicho, para ser exactos, tantas veces como pueda agotarse el diccionario. Pues, una vez que lo hayamos agotado la primera vez, podemos comenzar de nuevo con otra palabra y efectuar todo el recorrido de palabra a palabra en un orden diferente. Este juego de ensartar series de definiciones o comprehensiones, y de definiciones y comprehensiones de definiciones y comprehensiones, y de definiciones $\mathrm{y}$ comprehensiones de definiciones y comprehensiones de definiciones y comprehensiones, y así sucesivamente, no tiene necesariamente por qué terminar, ya que existen suficientes palabras en todo idioma para continuar durante todo el tiempo que, según han calculado los astrónomos, continuará la vida en la tierra y los planetas. Actualmente, este juego lleva unos cinco mil años, en el lenguaje ordinario y en la filosofía, pues el proceso de proseguir una comprehensión hasta sus implicaciones, y estas implicaciones hasta sus implicaciones y así sucesivamente, es el meollo del pensamiento analítico, y hay suficientes permutaciones de las palabras de un idioma para que pueda haber pensamiento analítico original durante otros diez mil millones de años. Hay, por ejemplo, en el Diccionario de la Real Academia unas cincuenta mil palabras, la mayor parte de las cuales significan conceptos analíticos. Podemos 
ordenar esas palabras en series de implicaciones cincuenta mil veces; y tomando series de implicaciones menos universales, es decir, de implicaciones que no agotan el diccionario sino únicamente partes de éste, llegaríamos a todas las permutaciones posibles de las cincuenta mil palabras, que son, en símbolos matemáticos ; 50000 factorial ó 50000 !, un número astronómico que significa $1 \times 2 \times 3 \times 4 \ldots \times 50000$; y podríamos hacer esto en $2^{50000} \mathrm{gru}-$ pos de palabras; un número supra-astronómico, si consideramos que $2^{64}$, el número de granos de trigo que el rey Shirham de la India "prometio" a su Gran Visir por la invención del ajedrez, es ya 18, 446, 744, 073, 709, 511, 615, y que el número de partículas en el universo no es más que $2^{263}$. Así, pues, prácticamente aun cuando no matemáticamente, es posible una infinidad de "sistemas" analíticos. ${ }^{8}$

Estos sistemas pueden referirse a una infinidad - una vez más prácticamente, no matemáticamente- de grupos de cosas, porque las extensiones de la totalidad de todos los conceptos analíticos son todas las cosas posibles. Así como la extensión del concepto "silla" son todas las sillas que son, han sido o serán, la extensión de cualquier concepto analítico - "manzana" "línea aérea", "universidad", etc., etc.- es la totalidad de todas las cosas así llamadas. La totalidad de tadas las extensiones, entonces, es la totalidad de todas las cosas posibles. Estas cosas están, en virtud de sus comprehensiones, agrupadas en clases, en "series", como lo están las comprehensiones mismas, pero las cosas no están interrelacionadas dentro de estas clases. Las comprehensiones analiticas determinan las propiedades que tiene cada miembro de la extensión o clase, pero no determinan las interrelaciones entre estos miembros. El concepto "silla" determina la clase de las sillas, pero no las interrelaciones entre las sillas. Una clase, pues, no es más que un agrupamiento, un conjunto de cosas que son comprehensionalmente similares; pero no constituye ningún orden extensional. ${ }^{\circ}$ El único orden conectado con la clase es el orden comprehensional de las implicaciones, y este orden, como hemos visto, es vago y arbitrario. Si existe una ley que ordene tales series de implicaciones, todavía no se la ha descubierto, a despecho de los enérgicos esfuerzos hechos a tal fin

8 Matemáticamente, $2^{50000}$ es sólo una parte infinitesimal de la infinidad. Desde el punto de vista de lo infinito, no hay diferencias entre $2,210000,250000,2100000$ o cualquier otro número finito. La infinidad matemática $\aleph_{0}$, sólo comienza después del último número contable. Según Leibniz, el número posible de proposiciones posibles es 107300000000000 (L. Couturat, Opuscules et fragments inédits de Leibniz, Paris, 1903; Vol. I, pág. 95).

9 Tiene, sin embargo, una interrelación significante, desarrollada por Donald Williams. Si las propiedades $\varphi$ y $\psi$ están presentes en una proporción dada entre los miembros de una clase, $v$. gr. $2 / 3$ de la clase son blancos y $1 / 3$ negros, entonces más subgrupos de la clase tienen $\varphi$ y $\psi$ en esta proporción que en cualquier otra. Inversamente, si en una parte observada de una clase están presentes las propiedades $\varphi$ y $\psi$ en una cierta proporción, entonces es calculable la probabilidad de la presencia de la misma proporción entre los miembros de la parte no-observada. (Véase Donald Williams, The Ground of Induction, Cambridge, Mass., 1947.) 
desde la Antigüedad.10 $\mathrm{Ni}$ el número exacto de conceptos que pueden quedar implicados en una comprehensión, ni su orden ni la ley de síntesis formada por todas las implicaciones de una comprehensión pueden determinarse actualmente. ${ }^{11}$ Dijimos, por consiguiente, que estas implicaciones se siguen una a otra en series. Aun cuando el orden es un poco más complicado que eso -consiste en series de series-, no es por ello más determinado. ${ }^{12}$ Es tan sólo un orden vago, como cúmulos de nubes, la una por encima y al lado de la otra: formaciones que todavía esperan al meteorólogo. Las moléculas de estas nubes de comprehensiones - que rodean, como "significados", a los conceptos analíticos- son los predicados, es decir, las palabras que constituyen el contenido de cada comprehensión.

Estos predicados, o sea los "miembros" de la comprehensión, carecen de interrelación exacta, al igual que los miembros de las extensiones. Tienen sus significados, pero esos significados a su vez son vagos, y también lo son los significados de esos significados, y así sucesivamente. De ahí la vaguedad del lenguaje cotidiano, y aún más, a causa de su mayor grado de abstracción, la vaguedad del lenguaje filosófico. El lenguaje analítico carece de la precisión del lenguaje científico. Este último utiliza conceptos cuyas comprehensiones son no-predicativas y relacionales. Las comprehensiones de los conceptos científicos o sintéticos, en otras palabras, no contienen predicados, sino relaciones formales.

Veremos en detalle lo que esto significa, pero por el momento bastará un ejemplo provisional. El concepto aristotélico del movimiento $\longrightarrow$ más bien del cambio- era un concepto analítico o filosófico. Consistía en relaciones analíticas pero no formales, es decir, en relaciones entre predicados: "realización de la potencia en cuanto potencia", "paso de la potencia al acto", "realización de lo móvil qua móvil", etc. De estos predicados Aristóteles derivó una multitud de consecuencias que, muy en detrimento del desarrollo intelectual de la hurnanidad, eran igualmente vagos que los conceptos originales. Tales

10 Especialmente de parte de Platón y Aristóteles, y de Kant. Cf. Fílebo, 16c-17a, la Metafísica de Aristóteles, Z XII, H VI; la Lógica de Kant y la Crítica del Juicio, Introd. Sec. V et passim. Obras centrales en esta fase de la filosofía clásica son las de Julius Stenzel, especialmente Gestalt und Zahl bei Platon und Aristoteles, Leipzig, 1924, y Nicolai Hartmann, "Aristoteles und das Problem des Begriffs" y "Zur Lehre vom Eidos bei Platon und Aristoteles", en Kleinere Schriften, II, Berlin, 1957. Una discusión detallada del problema en Kant se halla en Robert S. Hartman, "The Analytic and the Synthetic as Categories of Inquiry", en Perspectives in Philosophy, Ohio State University, 1953; págs. 55-78. Cf. Lewis White Beck, "Can Kant's Synthetic Judgments be made Analytic?", Kant-Studien, XLVII, 168-181 (1955/56). Véase también David García Bacca, Introducción a la Lógica Moderna, Barcelona, 1936; Introducción y Parte V.

11 Véase, sin embargo, como un intento de lograr tal determinación, Robert $S$. Hartman, op. cit., págs. 74 sigs.

12 García Bacca, op. ciț., págs. 229 sigs. También Hermann Lotze, Logik, Leipzig, 1912; págs. 46 sigs. Véase, sin embargo, el ingenioso ensayo de Lambert de estructurar la comprehensión analítica. García Bacca, op. cit,, págs. 27 sigs., C. I. Lewis, A Survey of Symbolic Logic, Berkeley, 1918; págs. 19 sigs. 
consecuencias fueron derivadas, más o menos, del mismo modo en que hemos dicho que se deriva una definición lexicográfica de otra; procedimiento cuya arbitrariedad se deja sentir aún, dos mil años después de Aristóteles, en lo que se tomó por ciencia - la filosofía natural - a lo largo de la Edad Media: en la alquimia, ${ }^{13}$ en la física aristotélica de la época de Galileo y, ya en pleno siglo xvir, en la teoría del flogisto de la química primitiva. ${ }^{14}$ Un ejemplo clásico de razonamiento analítico implicativo es el famoso argumento de Francesco Sizzi, jefe de los aristotélicos en Padua, contra el descubrimiento por Galileo de las lunas de Júpiter: "A los animales se les han concedido siete ventanas en el domicilio de la cabeza, que dejan pasar el aire al tabernáculo del cuerpo, para alumbrarlo, calentarlo y nutrirlo. ¿Cuáles son estas partes del microcosmos? Dos fosas nasales, dos ojos, dos oídos y una boca. De igual manera en el cielo, como en el microcosmos, hay dos estrellas favorables, dos que no son propicias, dos luminarias y Mercurio indeciso e indiferente. De ésta y de muchas otras similitudes en la naturaleza, tales como los siete metales, etc., que sería tedioso enumerar, colegimos que el número de planetas es necesariamente siete. Más aún, estos satélites de Júpiter son invisibles a

13 El razonamiento al parecer abstruso de los alquimistas encaja en un patrón consistente tan pronto se reconoce que tal razonamiento constituye implicaciones de conceptos analíticos.

14 El "flogisto" era un concepto típicamente analítico, que. Lavoisier convirtio en sintético mediante la introducción de relaciones cuantitativas en la comprehensión del concepto químico fundamental de "combustión". De tal suerte, hizo en la química lo que Galileo en la física. En su famosa "Tabla de sustancias simples que pertenecen a todos los reinos de la naturaleza y que pueden considerarse como los elementos de los cuerpos", Lavoisier constrastó los conceptos nuevos y los viejos:

Nombres nuevos

Luz

Calor

Oxigeno

Ãzoe (Nitrógeno)

Hidrógeno
Nombres viejos

Luz

Calor

Principio o elemento de calor

Fuego. Flúido ígneo

Materia de fuego y de calor

Aire deflogistizado

Aire empíreo

Aire vital $o$ base del aire vital

Aire o gas flogistizado

Mefitis, o su base

Aire o gas inflamable o la base del aire inflamable

(Lavoisier, Elementos de la química, Introducción a la Parte II.) Pero Lavoisier tuvo una visión menos clara de su sistema que Galileo. No vio que lo fundamentalmente nuevo en su procedimiento era el método cuantitativo - la ley de la constancia del peso a través de un cambio químioo-, y juzgó que su logro fundamental había sido su clasificación de los elementos químicos: un procedimiento de lógica analítica extensional. De ahí que llamara a esta clase de lógica, erróneamente, "la lógica de todas las ciencias" (Lavoisier, op. cit., Prefacio, par. 19). 
simple vista $y$, por lo tanto, no pueden ejercer influencia en la tierra $y$, por lo tanto, serían inútiles $\mathrm{y}$, por lo tanto, no existen. Además, los judíos y otras naciones antiguas, así como los europeos modernos, han adoptado la división de la semana en siete días y les han dudo nombre a ejemplo de los siete planetas. Ahora bien, si aumentamos el número de los planetas, todo este hermoso sistema se vendrá al suelo." ${ }^{15}$ Argumentos como éste nos parecen extraños hoy día sólo porque Galileo introdujo en la filosofía natural una manera muy diferente de razonar. El número 7 ha perdido hoy su poder implicativo, y argumentos como el que acabamos de citar parecen colecciones de sofismas. Pero en la filosofía moral esta clase de razonamiento abunda todavía, y no nos parece extraña.

Galileo define el concepto "movimiento" -que para él, como para Aristóteles, era el concepto central de la filosofía natural- de una manera muy distinta de la de Aristóteles: no analítica, sino sintéticamente, no mediante relaciones predicativas, sino formales. La relación que define al movimiento - más bien que al cambio- es " $\frac{s}{t} "$ - la relación entre el espacio recorrido por un cuerpo y el tiempo en que se efectúa el recorrido. Para nosotros, hoy, tal cosa es evidente: si un cuerpo recorre cien kilómetros en cinco horas, su "velocidad" es de veinte kilómetros por hora; pero para la época de Galileo esta reducción de algo que era un principio cósmico inherente tanto al mundo supralunar como al sublunar, al mundo de Dios y todas sus criaturas, a una división aritmética corriente, fue tan revolucionaria como para constituir una herejía. ${ }^{16}$ El cambio, principio teleológico del mundo, se convirtió en movimiento: una simple relación entre una distancia y un período de tiempo. ${ }^{17}$ Lo que los contemporáneos de Galileo no vieron, y lo que todavía hoy no reconocen todos los científicos y los filósofos de la ciencia como la médula del método científico, es que esta simplicidad se halla incrustada en una matriz de relaciones formales que incorpora al fenómeno en un contexto universal. El científico exacto, como Alicia en el país de las maravillas, atraviesa un espejo y entra en un mundo de formas puras, donde el mundo de la realidad concreta aparece

15 J. J. Fahie, Galileo, His Life and Work, London, 1903; pág. 103. El modelo aristotélico para esta clase de argumento puede hallarse, por ejemplo, en De Caelo, Libro II, Cap. 4. El argumento de Sizzi aparece en su Dianoia astronomica, 1610.

16 Ockham y otros que se habían anticipado a Galileo en la deshipostatización del movimiento, lo habian hecho filosofica y no científicamente, y por ello menos eficazmente que Galileo. Ellos no habían hecho que la Iglesia se opusiera, ni sustancialmente que la opinión pública favoreciera, a la nueva doctrina. Cf. H. Shapiro, Motion, Time and Place according to Ockham, Franciscan Institute Publications, Philosophy Series, $\mathrm{N}^{0}$ 13. También Franciscan Studies, XVI, 213-303 (septiembre de 1956), especialmente págs. 248 sigs.

17 Parte de la maravilla de la reducción galileana está capturada, en ropaje moderno, en Gerald Holton, Introduction to Concepts and Theories in Physical Science, Cambridge, Mass., 1952; págs. 1-6. Véase también Cap. 2, págs. 17-34 y Cap. 3, págs. 50 sig. 
transfigurado, transformado y en su esencia. ${ }^{18}$ En consecuencia, la simple relación galileana entre $s$ y $t$ pudo ser elaborada y aumentada, y todo un nuevo mundo pudo derivarse de ella: el mundo que hoy nos rodea. La fórmula de Galileo condujo así a una multitud de consecuencias; pero en lugar de ser implicaciones fortuitas, como las que se hallaban contenidas en el principio teleológico de Aristóteles, estas consecuencias fueron determinadas con precision, definidas con exactitud, deducidas y demostradas lógicamente, y verificadas empíricamente; y condujeron a los sistemas de Newton y Einstein, sistemas en el sentido estricto, basados no en conceptos analíticos materiales, sino en conceptos sintéticos formales, no en comprehensiones predicativas, sino relacionales.

La relación mutua entre la comprehensión y la extensión en los conceptos analíticos y los sintéticos, respectivamente, determina la diferencia lógica fundamental entre la filosofía y la ciencia: En los conceptos analiticos de la filosofía, la comprehensión y la extensión varian en proporción inversa, mien. tras que en los conceptos sintéticos de la ciencia varían en proporción directa. En los conceptos analíticos, cuantos más predicados están conténidos en la comprehensión, menos son los casos a los cuales es aplicable el concepto; y, a la inversa, cuantos más sean los casos a los cuales puede aplicarse el concepto, menos son los predicados incluidos en su comprehensión. Lo contrario sucede con los conceptos sintéticos: cuantas más relaciones están contenidas en la comprehensión, más son los casos a los que puede aplicarse el concepto, y cuantos más son los casos a los que puede aplicarse, más son las relaciones que están contenidas en su comprehensión. En otras palabras, la comprehensión analítica aumenta en el mismo grado en que la extensión aumenta, y viceversa. ${ }^{10}$ Esto es evidente si recordamos que la extensión es la aplicación de la comprehensión, y que la diferencia entre comprehensión sintética y comprehensión analítica es el carácter relacional de la segunda y la carencia de tal carácter de la primera. Obviamente, puesto que el concepto de clase surge por abstracción de las propiedades què tienen en común un grupo de cosas, mientras más sean estaş cosas, menos serán las propiedades que tienen en común, y, a la inversa, mientras menos sean estas cosas, más serán las propiedades que tengan en común. Una cosa individual tiene, obviamente, una infinidad de propiedades que no tiene ninguna otra cosa. De tal suerte, la comprehensión aumenta en el mismo grado en que el número de objetos clasifi-

18 Cf. los famosos libros de Mr. Tompkins por George Gamow, Mr. Tompkins in Wonderland ("Dedicado a Lewis Carrol y a Niels Bohr"), New York, 1940; Mr. Tompkins Explores the Atom, New York, 1947.

19 García Bacca, op. cit., pág. 239. Lo que nosotros llamamos "concepto analítico", García Bacca lo llama "concepto de abstracción total", y lo que nosotros llamamos "concepto sintético", él lo llama "concepto funcional". Cassirer llama al primero "sustancial", y al segundo, como García Bacca, "funcional". La regla de la variación inversa de la extensión y la comprehensión analíticas tiene algunas cualificaciones que son, sin embargo, impertinentes en nuestro contexto. 
cados disminuye, y viceversa. Es también obvio que hay más interrelaciones entre más cosas que entre menos cosas. Así, pues, si las interrelaciones están contenidas en una comprehensión, mientras más interrelaciones haya en una comprehensión, más serán las cosas interrelacionadas a que pueda aplicarse ésta. A la inversa, mientras más cosas interrelacionadas haya, más relaciones estarán contenidas en la comprehensión que se refiere a ellas.

Si bien en cualquier manual de lógica se examina la variación en proporción inversa a la comprehensión y la extensión de los conceptos analíticos, no ocurre lo mismo con la variación en proporción directa de los conceptos sintéticos. Sin embargo, esta variación es igualmente obvia y conspicua en el desarrollo de la ciencia. Así, pues, el progreso de Galileo a Newton fue un aumento de comprehensión y un aumento correspondiente en extensión del concepto galileano de "movimiento". Newton aumentó la comprehensión de

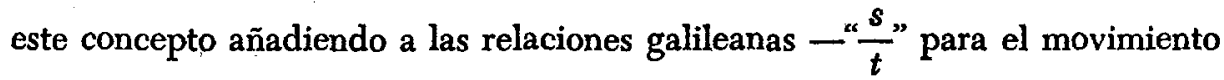

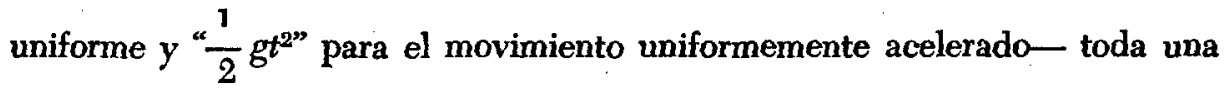
red de relaciones adicionales y más universales: las relaciones entre fuerza y cantidad de movimiento, cantidad de movimiento y velocidad, velocidad y masa, masa y densidad, densidad y volumen, etc. Las relaciones galileanas vinieron a ser así, para Newton, tan sólo dos entre muchas; fueron incorporadas en una red de relaciones, y esta red fue aplicable a muchas más cosas que la mecánica galileana. De tal suerte, Newton no sólo aumentó la comprehensión, sino también la extensión de los conceptos galileanos originales. Lo que es más, él aumentó esta extensión tanto cuantitativa como cualitativamente. Cuantitativamente, al incluir el inventario completo del mundo natural, desde los planetas hasta las mareas, y no sólo los cuerpos individuales, sino también las combinaciones de los cuerpos; cualitativamente, al extender su aplicación de los "casos ideales" de Calileo a los casos reales: definiendo las desviaciones de los fenómenos reales respecto a las fórmulas matemáticas del sistema. De tal suerte el aumento comprehensivo aumentó la extensión tanto cuantitativa como cualitativamente: aumentó el alcance y la exactitud de la aplicación. ${ }^{20}$

\section{La relación entre los conceptos analiticos y sintéticos}

La diferencia entre filosofia y ciencia es, entonces, la misma que hay entre el carácter lógico de los dos conceptos utilizados en las dos ramas del

20 Otra ilustración notable de esta relación es la diferencia en complejidad por una parte, $y$ en aplicabilidad por la otra, entre la geometría euclidiana y la cartesiana. (Véase Paul R. Halmos, "Innovation in Mathematics", en Scientific American, CXCIX, págs. 66-73, septiembre de 1958.) 
conocimiento. La ciencia utiliza conceptos de generalidad con precisión, en tanto que la filosofía utiliza conceptos de generalidad sin precisión. La precisión es lo que diferencia la comprehensión de las dos clases de conceptos. La comprehensión del concepto "movimiento" en Galileo es precisa, mientras que en Aristóteles es imprecisa. Los textos de lógica actuales tampoco nos ayudan a determinar con precisión el concepto de precisión. Es un concepto comprehensivo, y tenemos que remontarnos hasta Kant para hallar una discusión lógica de este concepto. Es el viejo problema pitagórico-platónico-aristotélico de la limitación, del ọ́ıúós: la demarcación de territorio, trátese de una porción de tierra o de un dominio del pensamiento. Kant fue uno de los últimos que lo investigaron dentro de un texto de Lógica. Los conceptos analíticos son para él, como para nosotros, los conceptos de la filosofía, en tanto que los sintéticos son los de la ciencia. Los primeros son dados, como los conceptos empíricos de la vida diaria, sólo que son dados más bien a priori - como, por ejemplo, el concepto "sustancia"- que a posteriori -como, por el ejemplo, el concepto "agua". 21 Los conceptos sintéticos o científicos, en cambio, son construidos por la mente humana, como, por ejemplo, el concepto geométrico "ćrrculo". La precisión de cualquier concepto consiste en la completa determinación de su significado en un mínimo de términos, "conceptus rei adaequatus in minimis terminis, complete determinatus". ${ }^{22}$ Los conceptos dados; sea a priori o a posteriori, no pueden tener determinación completa porque con ellos nunca se puede precisar si el concepto ha sido agotado. Puesto que el concepto es una cosa y aquello a lo que el concepto se refiere - la realidad concreta o trascendental de la cual el concepto es abstraído- es otra cosa distinta, esta última puede contener elementos que no han sido incluidos en el primero. Sólo con conceptos constructivos puede uno estar absolutamente seguro de que contienen todo lo que contiene su referente, pues estos conceptos construidos se originan junto con, y en realidad son, aquello a que se refieren. Poseen precisión completa, puesto que son creaciones de la propia mente humana más bien que abstracciones. De tal suerte Kant clarifica la diferencia entre la precisión posible para los conceptos analíticos o filosóficos y para los sintéticos o científicos.

Kant no clarifica en detalle en qué consiste exactamente la mayor precisión de los conceptos sintéticos, ni cuál es, en detalle, la relación lógica exacta entre las dos clases de conceptos. Pero tres resultados fundamentales se derivan de la Lógica de Kant, junto con observaciones que éste hace en la Teoría Transcendental de los Métodos en la Crítica de la Razón Pura: ${ }^{23}$ primero, que sí existen estas dos clases de conceptos; segundo, que existe

21 Obsérvese que éste es un concepto analítico a posteriori en el sentido de la Lógica. 22 Kant, Lógica, \$99. Para detalles, véase Robert S. Hartman, "The Analytic and the Synthetic as Categories of Inquiry", Perspectives in Philosophy, Ohio State University, Columbus, 1953.

23 A 713 sigs., "Disciplina de la razón pura en el uso dogmático". 
entre ellos esta diferencia fundamental de precisión; tercero, que dentro de la clase de conceptos analíticos existe una jerarquía de precisiones. Esta jerarquía comienza con la mera Descripción, que carece de toda precisión y "no tiene reglas y es únicamente material para la definición"; continúa en la Exposición, que es "la sucesión representativa de las propiedades del concepto descubiertas mediante el análisis"; y termina en la Definición, que es "el concepto completamente determinado en un mínimo de términos". Puesto que el concepto en cuestión aquí es el analítico, nunca puede ser completamente distinto y preciso, pues "uno nunca puede saber, mediante ninguna prueba, que todas las propiedades de un concepto dado han sido agotadas por el análisis. Por lo tanto, toda definición analítica debe considerarse incierta". ${ }^{24}$ De aquí la falta de precisión de los conceptos filosóficos; éstos no son sino una clase de conceptos dados, iguales a los conceptos empíricos de la vida diaria. Ellos no son, como lo han expresado diferentes filosófos de la ciencia moderna, ni más ni menos que abstracciones derivadas del sentido común. ${ }^{2 \bar{\sigma}}$

Los conceptos sintéticos o científicos no pueden ordenarse en una jerarquía de claridades porque ellos son transparentemente claros en razón de su propio origen. Ellos son "precisos desde el momento en que se hacen", en tanto que los conceptos analíticos o filosóficos "han de hacerse precisos" mediante el análisis. ${ }^{26}$ Cómo llega uno a los conceptos sintéticos, tales como los axiomas de las matemáticas, no lo dice Kant, aunque Galileo ya lo había dicho y los científicos y filósofos modemos nos lo repiten. ${ }^{27}$ La lógica de Kant nos da el fundamento, aunque no los detalles, de una lógica comprehensional. Nos da la diferencia entre los conceptos analíticos y los sintéticos y, por lo tanto, entre el método filosófico y el científico; y nos da la jerarquía de claridades analíticas que nos proporciona un análisis de precisión comprehensional más

24 Kant, Lógica, \$104.

25 Véase nota 5.

26 Kant, Lógica, Introducción, Sec. VIII.

27 A este punto se refieren los escritos sobre la fenomenología y la psicología de la creación, tales como Jacques Hadamard, The Psychology of Invention in the Mathematical Field, Princeton, 1945. La lógica del proceso ha sido discutida por Cassirer y algunos de sus seguidores. En Kant, la distinción entre lógica transcendental y lógica formal, epistemología y lógica, conduce a dificultades terminológicas. En los conceptos "sintéticos a priori" de las Matemáticas y la Física en la Critica, la parte a priori es lo que es sintético en términos de la Lógica. Estos conceptos, entonces, son a la vez epistemológica y lógicamente sintéticos. Los conceptos de la Metafísica de la Naturaleza, en la Crítica, son "sintéticos a priori" en un sentido diferente -lo a priori aquí es analítico a priori en el sentido de la Lógica kantiana. Estos conceptos, entonces, son epistemológicamente sintéticos y lógicamente analíticos a priori. En ambos casos, lo que es sintético de acuerdo con la Crítica es analítico a posteriori de acuerdo con la Lógica. De ahí que los conceptos de las Matemáticas y de la Física de la Crítica sean, en términos de la Lógica, sintéticos a priori y analíticos a posterion, y los de la Metafísica de la Naturaleza, en los mismos términos analíticos, tanto a priori como a posteriori. Para una versión moderna de lo analítico a posteriori véase José A. Benardete, "The Analytic A Posteriori and the Foundations of Metaphysics", Journal of Philosophy, LV, págs. 503-514 (junio 5, 1958). 
bien que extensional: una clarificación de Teilung (análisis) comprehensional más bien que Einteilung (división) clasificatoria. ${ }^{28}$ No nos clarifica el origen lógico de la precisión sintética o científica, ni su relación con la claridad analítica o filosófica. No nos ayuda a entender el proceso que transforma la precisión "incierta" de los conceptos analítico-filosóficos en la precisión "cierta" de los conceptos sintético-científicos: la logica del proceso que condujo de Aristóteles a Galileo, de la filosofía natural a la ciencia natural.

Para entenderlo debemos volvernos hacia la filosofía de Cassirer y sus sucesores. Entonces descubrimos que los conceptos sintéticos suceden a la jerarquía de precisión de los conceptos analiticos. La diferenciación del material dado, que conduce de la Descripción a la Exposición a la Definición, no se detiene en esta última. Más bien descansa en este punto-definición analítica- para dar el salto a la construcción sintética. El científico creador debe haber agotado el concepto analítico hasta el punto en que sea capaz de condensar incluso los minimi termini de la definición analítica en un solo término que, como un axioma, sirva de punto de partida para un sistema. En términos de una metáfora, este proceso podría ilustrarse como un faro cuya luz rotara sobre las aguas. La Descripción extrae de la oscuridad de lo desconocido - el fondo del mar- ciertos materiales que trae a la superficie y construye con ellos una base para el conocimiento, la base del faro. La Exposición selecciona, de esta base, aquellos materiales que tienen relación con el problema y construye una estructura más alta y más exacta. La Definición límita más aún el problema y, con un mínimo de materiales, coloca el techo de la edificación. La Construcción capta entonces la esencia única de toda la obra, la corona con el reflector, el axioma, al cual expande hasta convertirlo en un Sistema, el rayo de luz, y éste, a su vez, ilumina vastas y nuevas regiones de la oscuridad original.

Este procedimiento tiene implicaciones fenomenológicas y psicológicas que han sido ampliamente discutidas y no tienen por qué ser objeto de nuestra atención; pero sus implicaciones lógicas deben ser enunciadas.

La diferencia entre la precisión incierta de los conceptos filosóficos y la precisión cierta de los conceptos científicos es, según hemos visto, la diferente estructura de las comprehensiones de estos respectivos conceptos. La comprehensión del concepto analítico contiene en sí otros conceptos igualmente abstraídos, cada uno de los cuales representa una escala de abstracción de dimensiones potencialmente tremendas. Así, para referirnos otra vez al concepto aristotélico de movimiento, defínido como "la transición de la potencialidad a la actualidad", si queremos entenderlo debemos explicar los conceptos contenidos en él - “transición", "potencialidad", "actualidad"-, y subsiguientemente los conceptos contenidos en esas explicaciones, los conceptos contenidos en las explicaciones de las explicaciones, y así sucesivamente, hasta que 
llegamos a aquello de lo cual toda la cadena es el predicado, algún individuo cuya comprehensión analítica es la totalidad de las explicaciones dadas. La estructura exacta de este proceso no es conocida, y los intentos clásicos de "división" no son satisfactorios. ${ }^{29}$ Por lo tanto, dijimos que este procedimiento es similar a pasar de una definición del diccionario a otra. En el caso presente, el diccionario debería ser un diccionario filosófico. Al pasar de una definición a otra en él, cualquier lego inteligente podría escribir un tratado sobre el movimiento o sobre cualquier otro tema filosófico, una vez que se le haya dado una definición. Lógicamente, este proceso representa una especificación o deducción analítica, el proceso opuesto a la abstracción: el de extraer, a partir de un concepto dado, todos los conceptos contenidos en él, y de los contenidos de esos conceptos, los contenidos contenidos en ellos, y así sucesivamente -un proceso prácticamente infinito $\mathrm{y}$, por lo tanto, fundamentalmente diferente del de la deducción sintética, que es estrictamente regido por el sistema en cuestión.

La definición analítica, pues, es como un iceberg que sólo muestra su parte superior, mientras su mayor parte se halla bajo el agua. La definición analítica oculta el proceso de generalización que ha producido la cima. En consecuencia, Kant dice con mucha razón que la argumentación filosofica debería conducir $a$ la definicón y no partir de ella - como hace el método léxico. ${ }^{30}$ Para desarrollar un concepto filosófico, es preciso desarrollar todas las implicaciones de los conceptos contenidos en él. Mediante tal desarrollo - similar a la acción de pelar una cebolla tela a tela, o a la de encontrar una caja dentro de otra caja en un juego de cajas chinas- desarrollamos, precisamente, eso que se llama filosofía. Un "sistema" filosófico no es más que el desarrollo de un concepto analítico. ${ }^{31}$

Un sistema científico es muy diferente. El "contenido" de un concepto sintético no consiste en conceptos que contienen conceptos que contienen conceptos, y así sucesivamente. Consiste en términos que están relacionados con términos. El modelo de una comprehensión sintética es una red más bien que una cebolla o un nido de cajas chinas. Los conceptos de una comprehensión analítica no tienen, como hemos visto, ninguna relación intrínseca entre sí; y esto, precisamente, es lo que los hace logicamente iguales a los conceptos de la vida diaria. Los conceptos contenidos en la definición de "hombre" - “animal" y "racional"- no tienen una relación intrínseca entre sí, es decir,

29 Véase nota 10.

30 Critica de la Razón Pura, A 730 sig. Las matemáticas, en cambio, deberían empezar con la definición, pero esta definición es sintética. La definición analítica es, pues, el punto final de su argumento correspondiente -el filosófico-, en tanto que la definición sintética es el punto inicial del argumento que le corresponde -el matemático y científico. Por otra parte, la definición analítica es el punto inicial del método léxico, y la definición sintética es el punto final del pensamiento creador.

s1 Existe gran confusión en la filosofía acerca del significado de "sistema". Cf. G. E. Moore, "Wittgenstein's Lectures in 1930-1933", en Mind, LXIII, págs. 7 sigs. (1954). 
una relación que surja de su propio significado. El concepto "animal", como tal, no implica el concepto "racional", ni, a la inversa, el concepto "racional" implica el de "animal". Más bien, la relación entre los dos se basa en el referente de los dos conceptos, que es también el referente del concepto "hombre". Así, como Aristóteles observaba ya, ${ }^{32}$ la unidad de la definición (analítica) se basa en la entidad definida. Esto quiere decir que los conceptos contenidos en una definición analítica están interrelacionados verticalmente, por la totalidad de abstracciones que, partiendo de la entidad definida, ascienden hasta alcanzar tales conceptos, o, a la inversa, por la totalidad de especificaciones que, partiendo de tales conceptos, descienden hasta la entidad. La última cajita china, por decirlo así, es la entidad. Esta es, como Aristóteles sostuvo correctamente, inaccesible a la cognición (analítica), que comienza con la masa de propiedades que Kant llama descripción. La entidad a la cual ésta pertenece es, como tal, la unidad de esas propiedades; se la puede captar únicamente mediante una intuición no-discursiva. La última cajita china nunca se alcanza; continuamente nos acercamos a ella. En nuestro símil del faro, se halla en el fondo del mar. Cuando la imaginación creadora del científico salta de lo analítico a lo sintético, cuando instala la luz en la torre, antes debe haber buceado, por intuición, hasta el fondo de las profundidades. De otra suerte, la luz no tendrá fuerza, $y$, en lugar de iluminar las profundidades, jugueteará ociosamente en la superficie. Nuestra cognición (analítica) sólo profundiza hasta el nivel de la Descripción, la base del faro, las propiedades simples de las cosas. Una vez allí, sólo puede sondear las profundidades de las cuales se han extraído los materiales de la base; nunca puede, como cognición, bucear en esas profundidades. Para eso necesitamos un poder diferente. Para nuestra cognición, el iceberg de que hablamos antes tiene una profundidad infinita; la cebolla se hace pelar continuamente, su corazón es infinitamente pequeño.

De tal suerte, el pensamiento analítico es profundamente desalentador. Su única salvación es el pensamiento sintético. Este parece rehuir las profundidades; pero los contenidos de tales profundidades son extraídos y analizados mediante minuciosos métodos de microscopía y espectroscopía intelectual. El pensamiento sintético es, por decirlo así, la microquímica del pensamiento analítico. Lo que parece inaccesible para el pensamiento analítico se con-' vierte en un asunto de rutina para el pensamiento sintético.

Los términos de una definición sintética están interrelacionados horizontalmente, en un tejido de relaciones. No tienen profundidad, pero flotan sobre la profundidad. $^{33}$ La diferencia entre término y concepto consiste en que el

32 Metafísica, Z XII, H VI.

33 Por esta razón, a los creadores de sistemas se lés reprocha la facilidad con que resuelven problemas "profundos". Sagredo a Salviati en Dos nuevas ciencias, de Galileo: "Vos presentáis estas recónditas materias con demasiada evidencia y facilidad; esta gran facilidad hace que sean menos apreciadas que si se presentaran de una manera más abs- 
término no tiene comprehensión ni, ciertamente, extensión. Toda su significación se deriva de su posición dentro del sistema, de su interrelación con otros términos. ${ }^{34}$ El término es tan formal que no se refiere a nada: no es ni abstracto ni abstraido, es construido. La única "referencia" a la realidad que le incumbe es la aplicabilidad del tejido, del cual él forma parte, a una totalidad de fenómenos que se interrelacionan mediante esa aplicación, pero que no derivan ninguna individualidad de esa aplicación. El término, en otras palabras, no es un nombre que se refiere a algo, como el nombre propio "Sócrates" se refiere a Sócrates o como el nombre general "movimiento" de Aristóteles se refiere a los objetos que se mueven. Es una variable que puede ser aplicada a cualquier individuo que encaje en el tejido de interrelaciones en cuestión. Como hemos visto, en la ecuación para "movimiento" de Galileo, un nombre general -el "movimiento" de Aristóteles- fue transformado en una relación entre dos términos: la división aritmética $\frac{s}{t}$. En un sistema lógicamente superior, esta relación puede ser transformada en una expresión matemáticamente superior, como el "movimiento" galileano en los sistemas físicos subsecuentes. Además, un nombre propio puede ser transformado en una relación entre términos: cuando ese nombre propio es considerado como una infinidad de propiedades y la "infinidad" es interpretada en un sentido matemático, como un número transfinito. ${ }^{35}$

Transformemos, en vía de ejemplo, un nombre general y un nombre propio (o un concepto general y un concepto singular) en términos de un sistema: el concepto general "hombre" y el concepto singular "Sócrates". El concepto

trusa" (Galileo, Two New Sciences, trad. por H. Crew y A. de Salvio, Evanston, Ill., 1946; pág. 162). Salviati contesta al efecto de que la "profundidad" es tan sólo una coartada para el pensamiento falaz.

34 Véase Ernst von Aster, Die Philosophie der Gegenwart, Leiden, 1935; pág. 147.

${ }^{35} \mathrm{Al}$ individuo se le define entonces como la extensión de una comprehensión con contenido $N_{1}$. Tal extensión debe ser singular, pues $\aleph_{1}$ significa un continuo no-denumerable, es decir, una Gestalt. La particularidad, por otra parte, o la no-singularidad, presupone abstracción, es decir, denumerabilidad de las propiedades comprehensionales. Pues las propiedades comunes a dos cosas cuando menos, no pueden ser abstraídas excepto una a una, o conjunto a conjunto. La particularidad, pues, puede definirse como la extensión de una comprehensión de contenido $\aleph_{0}$. Si la extensión de una comprehensión de contenído 1 es dos cosas, entonces las dos forman un todo orgánico. La definición de un todo orgánico es entonces: una pluralidad extensiva con comprehensión de contenido $N_{1}$. Véase Robert $S$. Hartman, "Value Theory as a Formal System"; en Kant-Studien, L (1958-1959). Para una aplicación de las matemáticas transfinitas a la teoría del valor, véase Edwin Mitchell, A System of Ethics, New York, 1950; págs. 126 sigs., y Robert S. Hartman, "El conocimiento del valor: Teoría de los valores a mediados del siglo xx", Diánoia: Anuario de Filosofía, 1958; págs. 132 sigs. Para una discusión detallada véase el mismo autor. La medición del valor, de próxima aparición. Sobre la naturaleza de las operaciones finitas y transfinitas en la logica, véase García Bacca, op. cit., págs. 69 sigs. La conexión entre el valor, por una parte, y lo finito y lo infinito, por la otra, se halla, desde luego, en la base misma de la filosofía occidental. Véase Aristóteles, Metafísica, Libro A, Cap. 5, y Platón, Filebo. 
"hombre" se refiere a todos los hombres, Śccrates inclusive. Todo miembro de la clase de los hombres, Sócrates inclusive, satisface la definición analítica de "hombre", es decir, "animal racional". Pero al decir todo esto, no hablamos ni del hombre ni de Sócrates, no decimos nada acerca de la naturaleza o la evolución del hombre, o de la vida y actividades de Sócrates. Discutimos las interrelaciones lógicas de los términos "hombre" y "Sócrates". Podríamos expresar todo esto en un silogismo: "Todos los hombres son animales racionales. Ścrates es hombre. Por lo tanto, Sócrates es animal racional." Aquí, "hombre", "Sócrates" y "animal racional" no significan nada más que nudos en la red de relaciones lógicas que es el silogismo: son términos más bien que conceptos. El término "hombre" no se refiere a ningún hombre -no tiene extensión- ni significa nada -no tiene comprehensión-. ${ }^{30}$ Su único "significado" es la relación que guarda con otros términos. Igual es el caso en los términos "Sócrates" y "animal racional". Por lo tanto, todos estos términos pueden ser reemplazados por cualesquiera otros, con tal de que se mantengan las relaciones que guardan entre sí. Lo único que cuenta son esas relaciones. Así, también podríamos escribir: Todos los bípedos implumes son animales. racionales. Sócrates es bípedo implume. Por lo tanto, Sócrates es animal racional. Aquí hemos reemplazado el término "hombre" por "bípedo implume", pero hemos mantenido las relaciones. Del mismo modo pueden ser reemplazados todos los demás términos. En realidad, la forma del silogismo no necesita término alguno. Todos ellos pueden ser reemplazados por símbolos mientras se mantenga la forma del todo, el tejido de interrelaciones. Así, pues, todas las $S$ son $P . x$ es $S$. Por lo tanto, $x$ es $P$ es un silogismo tan válido como el primero, y por razones más lógicas, además. Y si también reemplazamos las palabras que indican las relaciones, "todas", "son", "es una", "por lo tanto", llegamos a la expresión simbólica del silogismo, “ $(x) x \in \mathrm{S}$. J. $x \in P^{\prime \prime}$. Estos términos, a su vez, pueden ser manipulados de acuerdo con sus propias leyes, originando así nuevas formas como " $(x) \varphi x \supset \psi x$ ", que elabora el silogismo original en nuevos aspectos sin tener que tomar en cuenta ese original: el propio simbolismo se hace cargo y hace desarrollarse al pensamiento de acuerdo con sus propias. leyes. Las relaciones formales, en virtud de su propia esencia, en virtud de ser relaciones, hacen avanzar el pensamiento, pues la forma del pensamiento es, precisamente, relacionante.

El concepto analítico predicativo, entonces, no es más que la primera etapa, todavía sensorial, del pensamiento. Debe ser superada por la segun-

36 Como sí lo hace el concepto analítico "hombre". Aun éste, desde luego, es más abstracto que los seres reales a los que se refiere. "Si yo digo: 'Conocí a un hombre', la proposición no es acerca de un hombre: éste es un concepto que no camina por las calles; sino que vive en el limbo nebuloso de los libros de lógica. Lo que conocí fue una cosa, no un concepto, un hombre real con un sastre y una cuenta bancaria o una cantina y una esposa borracha" (Bertrand Russell, The Principles of Mathematics, Cambridge, 1903; pág. 58). 
da etapa, la de la relación sintética: transición que se ha efectuado en la filosofía natural y que debe efectuarse en la filosofía moral. Galileo declaró una y otra vez que su pensamiento matemático le había permitido hacer avanzar el pensamiento sin referirse a la observación sensorial original. "El conocimiento de un solo hecho, adquirido mediante el descubrimiento de sus causas, prepara a la mente para entender y determinar otros hechos sin necesidad de recurrir al experimento, precisamente como en el caso actual, en el que, sólo mediante la argumentación, el autor prueba con certeza que el alcance máximo ocurre cuando la elevación es de $45^{\circ}$. El demuestra, así, lo que nuonca quiza ha sido observado en la experiencia, es decir, que de otros tiros, aquellos que no alcanzan o van más allá de los $45^{\circ}$ por cantidades iguales, tienen alcances iguales." ${ }^{37}$ Del mismo modo debemos liberar al pensamiento ético del "sentido común" y darle las alas para que se eleve a sus propios dominios. Sus actuales conceptos analíticos lo arrastran entre la masa de la observación sensorial. Sólo su transformación en relaciones sintéticas puede darle el impulso elevador necesario. Las corrientes intelectuales capaces de elevarlo están presentes - las relaciones sistemáticas de la axiológica- y lo han estado durante mucho tiempo. Platón, Leibniz ${ }^{38}$ y otros han sentido su brisa, pero ninguno hasta ahora se ha propuesto seriamente diseñar las estructuras aerodinámicas apropiadas a esas corrientes.

El paso decisivo es la transición del concepto al término, de las relaciones analíticas a las sintéticas. Incluso el término es sólo un sustituto, un arrendatario, un lugarteniente para el símbolo. El "significado" lógico del término es formal: el término no es otra cosa que un punto en el que las relaciones se cruzan. Todo su significado radica en el hecho de ese cruzamiento, las relaciones en cuestión. El punto de intersección contiene esàs relaciones, es el diferencial de la relación. Así, pues, hablando estrictamente, el silogismo, mientras contenga términos, no es una proposición lógica. Se convierte en tal sólo cuando los términos son reemplazados por símbolos. "Ninguna proposición de lógica puede mencionar ningún objeto particular. La enunciación "Si Sócrates es un hombre y todos los hombres son mortales, entonces Sócrates es mortal' no es una proposición de lógica; la proposición lógica de la cual la anterior es un caso particular, es: 'Si $x$ tiene la propiedad $\varphi$, y todo lo que tiene la propiedad $\varphi$ tiene la propiedad $\psi$, entonces $x$ tiene la propiedad $\psi$, no importa lo que sean $x, \varphi, \psi$.' La palabra 'propiedad', que ocurre aquí, desaparece de la enunciación simbólica correcta de la proposición, pero 'si-entonces', o

a7 Galileo, Two New Sciences, trad. por Henry Crew y Alfonso de Salvio, Evanston, 1946; pág. 265.

${ }_{88} \mathrm{La}$ convicción de que era posible una lógica del valor que abarcara los temas de la filosofía moral de la misma manera que las matemáticas abarcan los de la filosofía natural, inspiró a Leibniz su "Ciencia General" y su "Característica Universal". Ver su Prefacio a la Ciencia General y Hacia una Característica Universal, 1679. 
algo que sirva al mismo fin, permanece." ${ }^{30}$ Hablando menos estrictamente, desde luego, las proposiciones que contienen términos son proposiciones de lógica, con los términos tomando el lugar de los símbolos. Así, pues, "Australia es grande" es una proposición de geografía, pero "Australia es grande o Australia no es grande" es una proposición de lógica. ${ }^{40}$ En la primera, "Australia" es un concepto, en la segunda es un término.

La diferencia entre el concepto analítico y el sintético, entonces, consiste en que aquél es material en tanto que éste es formal. El concepto analítico es material en el sentido de que su comprehensión consiste en otros conceptos analíticos, cada uno de los cuales tiene significado en su propio contenido. El concepto sintético es formal en el sentido de que su comprehensión consiste en términos que no tienen significado en sí mismos sino en el sistema del cual forman parte. No cuentan por derecho propio; son cruzamientos de relaciones. Para usar un símil geométrico, el término es un punto conectado con otros puntos, el concepto es un volumen que contiene otros volúmenes que a su vez contienen otros volúmenes, y así sucesivamente ad infinitum. Con todo, el concepto analítico "material" se hace prácticamente impertinente en el grado en que se desarrolla, en tanto que el concepto sintético "formal" se hace prácticamente más y más pertinente en el mismo grado. Sabemos cuál es la razón lógica de ello: la diferencia en la relación extensional-comprehensional de los dos conceptos. Tratemos ahora de descubrir la razón genética: ¿Cuál es la conexión con la realidad que tiene el concepto sintético y no tiene el concepto analítico?

De la analogía entre un término como un punto, y un concepto analítico como un juego de volúmenes se deriva un profundo y detallado análisis de la relación entre el concepto analítico y el sintético, o, como podríamos decir, el concepto analítico y el término. ${ }^{41}$ Si el concepto es como un volumen que contiene volúmenes que a su vez contienen volúmenes, y así sucesivamente $a d$ infinitum, entonces esos volímenes deben hacerse más y más pequeños y deben acercarse infinitamente a un punto, y esto quiere decir: un término. Juegos infinitos de implicaciones analíticas, en otras palabras, se aproximan hasta un solo término como su límite. Aquí tenemos un significado profundo de la palabra "término" el de ser un límite del significado total de un conjunto total de conceptos - y el significado original del concepto "término" era "límite". ${ }^{2}$ pág. XI.

39 Bertrand Russell, The Principles of Mathematics, Introducción a la segunda edición,

40 Rudolf Carnap, Foundations of Logic and Mathematics, Chicago, 1939; pág. 12.

41 Puesto que el término es un elemento de un sistema, una vez que el término es dado reconociéndosele que tiene sentido, se da todo el sistema, y de ahí la comprehensión del concepto sintético; en cambio, cuando se da un concepto como parte de una comprehensión analítica, la comprehensión no está dada aún en absoluto.

42 Terminus es la traducción latina del griego ög05, límite, demarcación, definición, ögos eran originalmente los mojones que delimitaban un pedazo del terreno. Cf. Ortega y Gasset, La idea de Principio en Leibniz y la evolución de la teoría deductiva, Buenos Aires, 
En esta analogía el concepto científico es el límite ideal de la especificación más y más comprehensiva de los conceptos filosóficos. Realmente, como concluirá cualquiera que conozca la noción de límites ideales, el concepto científico es la especificación más y más comprehensiva.

Esto explica, de un solo golpe, el poder del concepto sintético. El sistema de términos que éste significa representa todo un dominio de fenómenos y sus infinitas posibilidades analíticas. Al mismo tiempo explica el procedimiento mediante el cual se puede arribar de los conceptos analíticos a los sintéticos: la concentración infinita del contenido analítico. Nuestro resultado une la psicología de la creación científica, como lo comprueban los testimonios de los científicos creadores, ${ }^{43}$ con las investigaciones teóricas en la relación entre el mundo del hecho sensorial y el mundo de símbolos que es la ciencia.

La analogía que hemos empleado, de una aproximación infinita de volúmenes a un punto, ha sido elaborada en un particular aspecto lógico-matemático por Alfred North Whitehead. Una generalización de su procedimiento nos da el resultado que acabamos de mencionar, $y$, así, la relación que buscamos entre concepto y término, entre pensamiento filosófico y pensamiento científico. El método de Whitehead es el de la "abstracción extensiva", basado en el principio de "convergencia a la simplicidad con disminución de la extensión". Se trata, fundamentalmente, de una aplicación de la relación entre el todo y la parte a la relación entre el mundo empírico del sentido común y el mundo construido de la ciencia. El primero consiste en "objetos intelectivos de la percepción", el segundo en "objetos intelectivos de la ciencia". Los segundos se derivan de los primeros como límites ideales de "conjuntos convergentes de objetos de inclusión", y ciertamente, como esos conjuntos. Para expresarlo con sencillez, Whitehead formula la relación de inclusión de un juego de cajas chinas como una relación lógica y deriva todas las consecuencias lógicas de esta formulación. Grupos de conjuntos de objetos temporales de inclusión convergen en, y definen, un momento; grupos de conjuntos de objetos espaciales de inclusión convergen en, y definen, un punto. "Momento" y "punto" son definidos como los grupos correspondientes de conjuntos convergentes. El procedimiento de Whitehead puede aplicarse también a los conceptos. Grupos de conjuntos de objetos conceptuales de inclusión convergen en, y definen, un término. De tal suerte los tres niveles de la ciencia que hemos hallado en Kant -en Whitehead el "primer objeto intelectivo de la percepción", el "segundo objeto intelectivo de la percepción" y el "objeto intelectivo de la ciencia"- se conectan mediante una relación lógica.

1958. Lo que Ortega y Gasset llama "término" es lo que nosotros, con Kant, llamamos defintción analítica.

43 Cf. Edison: "El genio consiste en $1 \%$ de inspiración y $99 \%$ de sudor." Newton: "Yo mantengo mi mente continuamente ocupada con el tema." Galileo: 'Yo me convertí min cuerpo que caía", etc. Cf. John Laird, "Synthesis and Discovery", Proceedings, Artutotelian Society, XIX, págs. 46-85 (1918-1919). También véase nota 27. 
El resultado es el que ya hemos mencionado, que los conjuntos de objetos conceptuales de inchusión convergen en un término. Podemos llamar este método el de la "abstracción intensiva" basado en el principio de "convergencia a la simplicidad con disminución de la comprehensión".

Este método tiene consecuencias de largo alcance para la lógica, la fenomenología y la axiología. En la lógica, el tratamiento clásico de los problemas es el de la división que conduce a la esencia. Puede demostrarse que este tren de pensamiento platónico-aristotélico corresponde a la interpretación del pensamiento de Whitehead que hemos dado. ${ }^{44}$ Más obvia es la conexión con la fenomenología. Nuestra interpretación del procedimiento de Whitehead revela que este procedimiento es idéntico al método de reducción fenomenológica. La estructuración del proceso de Wesensschau como lo da, por ejemplo, Nicolai Hartmann, presenta, precisamente, los rasgos salientes del método de "abstracción intensiva": la naturaleza limital del objeto ideal, la "concentración hacia un punto" (punktuelle Konzentration) que conduce hasta aquél, y el salto desde la totalidad de "elementos categoriales" que constituyen el Hinleitung hasta el propio Schau inmediato, en un acto sui generis, que abstrae de, y sin embargo representa, esta totalidad. ${ }^{45}$

El término así alcanzado no es, desde luego, cualquier término dentro de un sistema, sino el término del sistema, el término de términos a partir del cual se originan el propio sistema y todos sus términos. Es la matriz del sistema, el axioma.

Esta expresión griega, pues, que significa aquello que vale la pena ser pensado, es el límite, en el sentido matemático preciso, de una totalidad infinita de contenidos analíticos. El mérito del pensamiento, lo que da al pensamiento su valor intrínseco, es esta con-centración, esta com-prehensión de una multitud infinita de contenidos categoriales, empíricamente abstraídos, en un solo punto infinitamente remoto de toda abstracción. Es la construcción: el significado intrínseco de toda abstracción. El axioma, pues, es de una dimensión diferente de la categoría, en el sentido matemático exacto de que un axioma vale por una infinidad de categorías. La comprehensión de un axioma, un

44 Para detalles ver el estudio del propio autor, de próxima aparición: La estructura de la definicion. Ver Alfred $\mathrm{N}$. Whitehead, The Concept of Nature, Cambridge, 1955; Caps. III-V y The Aims of Education, Cap. 9. También L. Susan Stebbing, A Modern Introduction to Logic, London, 1948; Cap. XXIII, y C. D. Broad, Scientific Thought, London, 1952; Cap. I. El símil del juego de cajitas chinas se encuentra en The Concept of Nature, pág. 61. Sobre un procedimiento similar de Leibniz -el ordenamiento de lo desconocido mediante el ordenamiento de lo conocido con la ayuda de enfoques infinitos-, véase "Letter of Mr. Leibniz on a General Principle Useful in Expaining the Laws of Nature", 1687, Loemker, L. E., ed., Leibniz: Philosophical Papers and Letters, Chicago, 1956; págs. 538 sigs.

45 Nicolai Hartmann, Grundzüge einer Metaphystk der Erkenntnis, Berlin, 1949; Caps. 64-70; en particular, págs. 514 sigs., 531 sigs. 
sistema sintético 0 una ciencia, vale por una infinidad de comprehensiones de una categoría, conjuntos de conceptos analíticos o una filosofía.

Con el axioma comienza el proceso de síntesis axiomática que es la ciencia.

Robert S. Hartakan 